\title{
ON THE JACOBI EQUATION AND MANIFOLDS WITH MULTIPLE CONJUGATE POINTS
}

\author{
J. M. Burns, E. Staunton, D. J. Wraith
}

\begin{abstract}
We investigate the phenomenon of multiple conjugate points along a geodesic. In the first instance, we investigate conjugate points in the context of the Jacobi equation, a second order ordinary differential equation, which captures precisely the geometry of conjugate points on surfaces. We then construct geometric examples which exhibit similar properties in higher dimensions.
\end{abstract}

\section{$\S 1$ Introduction.}

In this note we will consider the following Jacobi equation:

$$
y^{\prime \prime}(t)+K(t) y(t)=0
$$

where $t \in \mathbb{R}$ and $y(t)$ and $K(t)$ are real-valued functions with $K$ assumed smooth. The motivation for studying this equation arises from its geometric significance. This begins with the calculus of variations on surfaces, which we will now explain.

Consider a smooth curve $c(t), t \in[a, b]$, on some smooth surface $M^{2}$, joining points $P=c(a)$ and $Q=c(b)$. By a variation of $c(t)$, we will mean a smooth map

$$
H:[a, b] \times(-\epsilon, \epsilon) \rightarrow M
$$

such that $c(t)=H(t, 0)$ for all $t \in[a, b]$. Such a variation is called a homotopy if in addition we have $H(a, s)=P$ and $H(b, s)=Q$ for all $s \in(-\epsilon, \epsilon)$. We can view any variation as a one-parameter family of curves $c_{s}(t):=H(t, s)$ parametrized by $s \in(-\epsilon, \epsilon)$. The length function $L(s)$ for this one-parameter family of curves is differentiable, and a basic result in the calculus of variations states that $L^{\prime}(0)=0$ for every homotopy of $c(t)$ if and only if $c(t)$ is a geodesic. The 'second variation formula' describes the second derivative $L^{\prime \prime}$, and can be used to determine whether a geodesic is unstable/stable, in the sense that there is/is not a homotopy which descreases length. It turns out that the second variation formula involves the Gaussian curvature of the surface. Intuitively, it is not difficult to see that the appearance of curvature here is reasonable. For example, on the positively curved round sphere, a rubber band stretched along the equatorial great circle will contract if disturbed slightly, whereas it will not if stretched along the smallest equatorial circle on a catenoid (such as a power station cooling tower), which is a classic example of a surface with negative Gaussian curvature.

2010 Mathematics Subject Classification: 34C40, 53C22.

Keywords: conjugate points, geodesics, Jacobi equation, Riemannian manifolds 
Now suppose $c(t), t \in \mathbb{R}$, is a geodesic, and consider a variation through geodesics so that $c_{s}(t)$ is a geodesic for each $s \in(-\epsilon, \epsilon)$. Differentiating with respect to the variation parameter $s$ produces a vector field

$$
V(t):=\frac{\partial}{\partial s} H(t, s)
$$

along $c(t)$, called a Jacobi vector field. Suppose that $V(0)=0$, and let $P:=c(0)$. If $V(t)$ is everywhere orthogonal to $c(t)$ then it is clear that $V(t)$ is completely determined by a (vector length) function $y: \mathbb{R} \rightarrow \mathbb{R}$. Jacobi discovered that the function $y(t)$ satisfies the Jacobi equation $(J)$ subject to the initial conditions $y(0)=0, y^{\prime}(0)=\left|V^{\prime}(0)\right|$, and where the function $K(t)$ is the Gaussian curvature of the surface at the point $c(t)$. It is not difficult to show that $V(t)$ is orthogonal to $c^{\prime}(t)$ if, for example, there exists some $t_{1} \neq 0$ for which $V\left(t_{1}\right)=0$. In this case, the point $Q:=c\left(t_{1}\right)$ at which $V(t)$ vanishes is said to be conjugate to the point $P=c(0)$, at which $V(t)$ also vanishes.

In terms of the equation $(J)$ we define a point $t_{1} \in \mathbb{R}$ to be conjugate to some $t_{0} \in \mathbb{R}$ if there exists a non-trivial solution $y$ of $(J)$ for which $y\left(t_{0}\right)=y\left(t_{1}\right)=0$. If there is no solution with two zeros, then $(J)$ is said to have no conjugate points.

For surfaces of constant curvature, it is easy to solve the Jacobi equation explicitly: for example if the initial conditions are $y(0)=0$ and $y^{\prime}(0)=1$, we have that if $K \equiv$ $k>0$ then $y(t)=(1 / \sqrt{k}) \sin (t \sqrt{k})$; if $K \equiv 0$ then $y(t)=t$; and if $K \equiv k<0$ then $y(t)=(1 / \sqrt{-k}) \sinh (t \sqrt{-k})$. Thus in the case of constant positive curvature $k$ we obtain conjugate points at a separation of $\pi /(2 \sqrt{k})$, however in the case of non-negative curavture, we clearly have no conjugate points.

Note that there is one situation in which the conjugate points described by the equation $(J)$ and those on the surface behave differently: the case of a geodesic loop. Consider, for example, any great circle on a standard round sphere. This is a geodesic $\gamma: \mathbb{R} \rightarrow S^{2}(1)$ (assuming a unit speed parametrization). It is straightforward to see that for $(J)$, every point in the set $\{\pi n \mid n \in \mathbb{Z}-\{0\}\}$ is conjugate to $0 \in \mathbb{R}$. On the other hand, on $S^{2}$, there is only one point conjugate to $\gamma(0)$, namely $\gamma(\pi)$, since all other points $\gamma(\pi n)$ for $n \in \mathbb{Z}$ conincide with either $\gamma(0)$ or $\gamma(\pi)$. With a view towards geometry, when counting conjugate points we will adopt the convention that we count points on the manifold, that is, without multiplicity.

Consider a geodesic from any point on a surface. Locally, this will minimize distance from the initial point. This means that the geodesic represents the shortest path between the initial point and any subsequent point which lies on the geodesic, provided this distance is not too great. More precisely, any geodesic will minimize distance up to, but no further than the first conjugate point. This is the basic geometric significance of conjugate points, and is the starting point for many geometric and topological theorems. We can actually say more [Ch; p108]: if a geodesic from $p$ to $q$ minimizes distance as far as, but no further than $q$, then $q$ is either conjugate to $p$, or is the mid-point of a geodesic loop from $p$.

Note that surfaces with no conjugate points have been extensively studied, see for example $[\mathrm{BBB}],[\mathrm{BK}],[\mathrm{G}],[\mathrm{GG}]$. In terms of the equation $(J)$, a basic result about the non-existence of conjugate points is as follows: 
Theorem 1.1. [H; p351] Every non-zero solution of a Jacobi equation has at most one zero if and only if there is a solution which never vanishes.

Theorem 1.1 is the motivating result for this paper. We ask: can anything similar be said if there is more than one conjugate point? Although this question seems natural, to the best of our knowledge no study of multiple conjugate points appears in the literature, either in terms of the Jacobi equation or in terms of geometric realizations. We address the first of these issues in Theorem 1.2 below, and the second in Theorem 1.3.

Theorem 1.2. Let $S \backslash\{0\}$ denote the set of non-zero solutions to a Jacobi equation. Then either

(i) all solutions have infintely many zeros, or

(ii) there is an $n \in \mathbb{N}$ such that every solution has either $n$ or $n-1$ zeros, with both these possibilities occuring.

It should be noted that part (i) and the first part of (ii) in Theorem 1.2 are actually elementary consequences of the Sturm Separation Theorem, and thus are not new as such. The new content in Theorem 1.2 is the claim that when the maximum number of zeros for non-trivial solutions to a Jacobi equation is $n$, there must also be a solution with precisely $n-1$ zeros. This Theorem also raises the question of the existence, for each $n \in \mathbb{N}$, of Jacobi equations for which all non-trivial solutions have $n$ or $n-1$ zeros. Similarly we can ask for Jacobi equations for which the solutions have infinitely many zeros. In $\S 2$ we give examples to illustrate all of these cases.

In higher dimensions the geometry is more complicated. On any Riemannian manifold we can still consider a variation through geodesics, and obtain a Jacobi field $V(t)$ in the same way along the central geodesic $c(t)$. However, $V(t)$ is no longer determined by a single real-valued function. We obtain a Jacobi equation similar to $(J)$, but this time it takes the form

$$
\nabla_{c^{\prime}(t)}^{2} V+K\left(c^{\prime}(t), V(t)\right) V=0
$$

where $\nabla^{2}$ denotes the second covariant derivative of the vector field $V$ in the direction $c^{\prime}(t)$, and $K\left(c^{\prime}(t), V(t)\right)$ denotes the sectional curvature of the tangent plane spanned by $c^{\prime}(t)$ and $V(t)$. Conjugate points are defined in the analogous manner in terms of the vanishing of the vector field $V$, and the results about distance minimizing properties of geodesics continue to hold in this more general setting. However solving this Jacobi equation - now a vector equation - is not so easy. On the other hand, given upper or lower bounds on the sectional curavture, one can make some general comparison statements. The classical Rauch Theorem, for example, gives upper respectively lower bounds on the lengths of certain Jacobi fields in the presence of lower respectively upper bounds on the curvature, see [doC; page 210].

Although it is not difficult to find Jacobi equations which illustrate the various situations described by Theorem 1.2, constructing Riemannian manifolds which geometrically realize this multiple conjugate point behaviour is another issue. Our second main result addresses this: 
Theorem 1.3. Let $n \in \mathbb{N} \cup\{0, \infty\}$ and $d \in \mathbb{N}, d \geq 2$.

a) If $n$ is finite then on any smooth compact manifold $M$ of dimension $d$ there exists a Riemannian metric $g$, a point $x \in M$, and a g-geodesic $\gamma$ passing through $x$, such that there are at least $n$ points along $\gamma$ conjugate to $x$.

b) There exists a Riemannian metric on $\mathbb{R}^{d}$ for which there is a geodesic $\gamma$ passing through some point $x \in \mathbb{R}^{n}$, such that there are precisely $n$ points along $\gamma$ conjugate to $x$.

In the light of the above discussion, it is natural to ask about the conjugate point phenomona occurring on a given complete Riemannian manifold as a whole, as opposed to along a specific geodesic. We insist on completeness here as this ensures that the domain of every geodesic can be taken to be the whole of $\mathbb{R}$, thus each geodesic can be viewed as a smooth map $\gamma: \mathbb{R} \rightarrow M$. With this in mind, define the set $S \subset \mathbb{N} \cup\{0, \infty\}$ to be the set of extended natural numbers $n$ such that there is a geodesic $\gamma$ on $M$ and a point $\gamma\left(t_{0}\right) \in M$ which is conjugate to precisely $n$ other points along $\gamma$. We might call $S$ the conjugate spectrum of the manifold. It is easy to see that for a standard round sphere, the conjugate spectrum is precisely $\{1\}$, as all geodesics are closed loops. For any manifold of non-positive sectional curvature, the spectrum is $\{0\}$. Thus it makes sense to ask the following very general

Questions. What can be said about the conjugate spectrum of a Riemannian manifold? In particular, given a subset $S \subset \mathbb{N} \cup\{0, \infty\}$, can we find a Riemannian manifold $M$ which realizes this spectrum? How does curvature influence the possible spectra?

For the record, there are other interesting phenomena with topological and geometric significance that one can study along geodesics besides conjugate points. For example, one can study the way in which the mean curvature of distance spheres centred on the point $\gamma(0)$ evolves along $\gamma(t)$. This is closely related to the study of conjugate points. Instead of the equation $(J)$, one has a Riccati inequality which describes the evolution. This inequality relates the mean curvature of distance spheres to the Ricci curvature of the manifold. See for example $[\mathrm{W}]$.

Our third main result concerns the derivatives of solutions to Jacobi equations, and is analogous to Theorem 1.1.

Theorem 1.4. Suppose that the function $K$ in the Jacobi equation $(J)$ is nowhere zero. If every non-zero solution of $(J)$ has a derivative which vanishes at most once, then there exists a solution for which the derivative never vanishes.

This paper is laid out as follows. In $\S 2$ we prove Theorem 1.2. In $\S 3$ we construct examples which establish Theorem 1.3. Finally, in $\S 4$ we turn our attention to the derivatives of solutions to the Jacobi equation and prove Theorem 1.4.

The authors would like to thank A. Lytchak and G. Thorbergsson for helpful conversations during the preparation of this paper. 


\section{$\S 2$ The proof of Theorem 1.2.}

Lemma 2.1. If $f(t)$ is a non-zero (i.e. not identically zero) solution of a homogeneous second-order linear ODE, and $f\left(t_{0}\right)=0$ for some $t_{0}$, then $f(t)$ changes sign through $t_{0}$.

Proof. Suppose otherwise. Then we must have $f\left(t_{0}\right)=f^{\prime}\left(t_{0}\right)=0$. But this means that $f$ is the zero solution, giving a contradiction.

Let $S$ denote the set of solutions to a homogeneous second order linear ODE. It is well-known that $S$ is a two dimensional vector space, and as such, has a natural topology obtained by pulling back the standard topology on $\mathbb{R}^{2}$ by a linear isomorphism. With respect to this topology, we consider a continuous path $f_{s}$ in $S \backslash\{0\}$, where $s \in I$ is the path parameter and $I$ an interval. Each $f_{s}(t)$ is at least a $C^{2}$ function of $t$, and in particular is continuous. We will be concerned with the number and location of the zeros of $f_{s}$ as $s$ varies.

Lemma 2.2. Suppose that $f_{s_{0}}\left(z_{0}\right)=0$ for some $s_{0} \in I, z_{0} \in \mathbb{R}$. Then for any sequence $s_{n} \in I$ with $s_{n} \rightarrow s_{0}$, and for any choice of $\delta>0$, there is an $N \in \mathbb{N}$ such that for all $n>N, f_{s_{n}}(t)$ has a zero in the interval $\left(z_{0}-\delta, z_{0}+\delta\right)$.

Proof. First note that as $s_{n} \rightarrow s_{0}$, the functions $f_{s_{n}}$ converge pointwise to $f_{s_{0}}$. Now suppose the lemma is false, that is, there is a $\delta>0$ and a sequence $s_{n} \in I$ with $s_{n} \rightarrow s_{0}$ such that $f_{s_{n}}(t) \neq 0$ for all $t \in\left(z_{0}-\delta, z_{0}+\delta\right)$ and for all $n \in \mathbb{N}$. Pick $t_{1} \in\left(z_{0}-\delta, 0\right)$ and $t_{2} \in\left(0, z_{0}+\delta\right)$. By Lemma 2.1, either $f_{s_{0}}\left(t_{1}\right)>0$ and $f_{s_{0}}\left(t_{2}\right)<0$, or $f_{s_{0}}\left(t_{1}\right)<0$ and $f_{s_{0}}\left(t_{2}\right)>0$. If $n$ is sufficiently large we must therefore have $f_{s_{n}}\left(t_{1}\right)$ and $f_{s_{n}}\left(t_{2}\right)$ with opposite signs. As each $f_{s}$ is a continuous function, by the Intermediate Value Theorem each $f_{s_{n}}$ must have a zero in the interval $\left(t_{1}, t_{2}\right) \subset\left(z_{0}-\delta, z_{0}+\delta\right)$, giving a contradiction.

Corollary 2.3. With $f_{s}$ as above, suppose that $f_{s_{0}}$ has a zero at $t=z_{0}$. Then there is a continuous path $z_{s}, s \in\left(s_{0}-\epsilon, s_{0}+\epsilon\right)$ for some $\epsilon>0$, such that $f_{s}\left(z_{s}\right)=0$.

Proof. It clearly suffices to show that we can choose $z_{s}$ for $s$ sufficiently close to $s_{0}$ so that we have continuity at $s=s_{0}$. By Lemma 2.2, for any $\delta>0$, we can choose some zero in the interval $\left(z_{0}-\delta, z_{0}+\delta\right)$ for all $s$ sufficiently close to $s_{0}$. If no path can be chosen to be continuous at $s_{0}$ then there must be a $\delta^{\prime}>0$ and a sequence $\left\{s_{n}\right\}$ converging to $s_{0}$ such that $f_{s_{n}}$ has no zero in $\left(z_{0}-\delta^{\prime}, z_{0}+\delta^{\prime}\right)$ for all $n$. But this contradicts Lemma 2.2, hence the result.

Corollary 2.4. With $f_{s}$ as above, suppose that $I$ is a compact interval, and that for every $s \in I, f_{s}$ has precisely $n$ zeros $z_{s}^{1}<\ldots<z_{s}^{n}$. Then there exists an $\epsilon>0$ such that for all $i=1, \ldots, n-1$, we have $z_{s}^{i+1}-z_{s}^{i}>\epsilon$.

Proof. The zeros of any $f_{s}$, being finite in number are necessarily separated. By Corollary 2.3 , this means that for each $i=1, \ldots, n$, the zeros $z_{s}^{i}$ form a continuous path with respect to $s$. By assumption, no two adjacent paths $z_{s}^{i}$ and $z_{s}^{i+1}$ meet as $s$ varies, and so by the compactness of the $s$-domain $I$, we obtain a non-zero minimum separation between adjacent zeros. 
Corollary 2.4 says that under the given hypotheses, zeros cannot 'give birth' to multiple zeros as $s$ varies, nor can zeros come together to coincide. In conjunction with Corollary 2.3 , we further see that zeros cannot spontaneously appear or disappear, and thus each zero remains isolated and continuously varying with $s$.

Proposition 2.5. For any $f \in S \backslash\{0\}$, there is no continuous path $f_{s}$ in $S \backslash\{0\}$ with $s \in[0,1]$, such that $f_{0}=f$ and $f_{1}=-f$ with the property that each $f_{s}$ has precisely $n$ zeros, for some $n \in \mathbb{N}$.

Proof. Suppose otherwise. With the notation of Corollary 2.4, set $Z(s):=\sum_{i=1}^{n}\left|z_{s}^{i}\right|$. Then for each $s \in I, f_{s}(Z(s)) \neq 0$ since $Z(s)>z_{s}^{n}$. Moreover, since $z_{s}^{i}$ is continuous for each $i$ we see that $Z(s)$ is also continuous. Consequently, the composition $f_{s}(Z(s))$ is continuous with respect to $s$. But $f(Z(0))=f_{0}(Z(0))$ and $-f(Z(0))=f_{1}(Z(1))$ are both non-zero and with opposite signs, which contradicts the Intermediate Value Theorem given that $f_{s}(Z(s))$ is never zero. Thus no such path can exist.

We now specialise to the case where our second order linear ODE is of Sturm type, that is, takes the form

$$
p(t) y^{\prime \prime}+p^{\prime}(t) y^{\prime}+q(t) y=0
$$

for some functions $p(t)$ and $q(t)$. As above, let $S$ denote the solution set to this differential equation. We will need the following Sturm Separation Theorem (see [H; p335]):

Theorem 2.6. Assume that the functions $p(t)$ and $q(t)$ in the Sturm equation above are continuous on some interval $I \subset \mathbb{R}$. Let $f_{1}$ and $f_{2}$ be linearly independent solutions of this equation. Then the zeros of $f_{1}$ separate and are separated by those of $f_{2}$.

From this we obtain:

Corollary 2.7. Consider the set $S \backslash\{0\}$ of non-zero solutions to a Sturm equation. Then if some $f \in S-\{0\}$ has infinitely many zeros then so does every solution. On the other hand if $f \in S-\{0\}$ has $n$ zeros for some $n \in \mathbb{N}$, and $n$ is the maximum number of zeros of any function in $S-\{0\}$, then every solution has either $n$ or $n-1$ zeros.

Combining Corollary 2.7 with Proposition 2.5 immediately gives:

Theorem 2.8. If $n \in \mathbb{N}$ is the maximum number of zeros for any solution of a given Sturm equation, then there is also a solution with precisely $n-1$ zeros.

Given that the zeros move continuously, it is natural to ask about the circumstances under which the number of zeros drops from $n$ for $s<s_{0}$ say, to $n-1$ at $s=s_{0}$. It is not difficult to see that the only possible situation in which this can happen is if $z_{s}^{1} \rightarrow-\infty$ or $z_{s}^{n} \rightarrow \infty$ as $s \rightarrow s_{0}^{-}$. It is also clear that $z_{s}^{1} \rightarrow-\infty$ and $z_{s}^{n} \rightarrow \infty$ cannot both occur simultaneuously, as this would result in a solution with $n-2$ zeros, contradicting Corollary 2.7. The reason why these two possibilities cannot happen together can be seen from the Sturm Separation Theorem: if for some $f_{s_{0}}$ and $f_{s_{1}}$ we have $n$ zeros, and if $z_{s_{0}}^{i}>z_{s_{1}}^{i}$ for some $i$, then in order for the separation property to hold we must have $z_{s_{0}}^{i}>z_{s_{1}}^{i}$ for all $i$. In other words, all zeros must move in the same direction. So if $z_{s}^{n} \rightarrow \infty$ say, we cannot have $z_{s}^{1} \rightarrow-\infty$, and similarly in the converse.

The following provides a converse to Theorem 2.8 in the case $n=1$ : 
Theorem 2.9. ([H; p351]) Every non-zero solution of a Sturm equation has at most one zero if and only if there is a solution which never vanishes.

In summary we have:

Theorem 2.10. Let $S \backslash\{0\}$ denote the set of non-zero solutions to a Sturm equation. Then either

(i) all solutions have infintely many zeros, or

(ii) there is an $n \in \mathbb{N}$ such that every solution has either $n$ or $n-1$ zeros, with both these possibilities occuring.

If we now observe the that Jacobi equation $(J)$ is a special case of the Sturm equation, then Theorem 2.9 gives Theorem 1.1 of the introduction, and Theorem 2.10 immediately implies Theorem 1.2.

It is not difficult to construct examples of Jacobi equations to illustrate that both (i) and (ii) in Theorem 2.10 can occur.

Example 2.11. Consider the Jacobi equation

$$
y^{\prime \prime}+y=0
$$

The solution set is spanned by $\cos t$ and $\sin t$, and clearly every non-zero solution has infinitely many zeros.

Example 2.12. Consider the Jacobi equation

$$
y^{\prime \prime}-y=0 \text {. }
$$

The solution set is spanned by $e^{t}$ and $e^{-t}$ which are both non-vanishing, but also contains $\sinh t$ which vanishes at $t=0$.

Example 2.13. For any $n \in \mathbb{N}$, we construct functions $K(t)$ and $f(t)$ such that $f(t)$ is a solution of the Jacobi equation

$$
y^{\prime \prime}+K(t) y=0
$$

which has precisely $n$ zeros. As a preliminary, consider the piecewise linear function $k(t)$ defined to be 1 for $t \in[-1 / 10, n \pi+1 / 10]$, and equal to -1 for all other $t \in \mathbb{R}$. Now smooth $k(t)$ to a function $K(t)$ in the obvious way over the intervals $(-1 / 10-\epsilon,-1 / 10)$ and $(n \pi+1 / 10, n \pi+1 / 10+\epsilon)$ for some very small $\epsilon>0$. Consider the solution $f(t)$ to the resulting Jabobi equation with initial conditions $f(0)=0, f^{\prime}(0)=1$. Clearly, we must have $f(t)=\sin t$ for $t \in[-1 / 10, n \pi+1 / 10]$, and $f(t)$ has precisely $n$ zeros in this interval. On each of the intervals $(-\infty,-1 / 10-\epsilon)$ and $(n \pi+1 / 10+\epsilon, \infty), f(t)$ takes the form $f(t)=c_{1} e^{c_{2} t}$ for some constants $c_{1}$ and $c_{2}$. (The pair of constants for one of the intervals will most likely be different from the pair for the other interval.) If $c_{1} \neq 0$ then the function $c_{1} e^{c_{2} t}$ is never zero. The fact that $f(-1 / 10)$ and $f(n \pi+1 / 10)$ are both non-zero means that provided $\epsilon$ is sufficiently small, $f$ will not have any zeros on either of the smoothing intervals and consequently the values for $c_{1}$ will both be non-zero. Thus overall, $f(t)$ will have precisely $n$ zeros. 


\section{$\S 3$ Geometric realizations.}

The aim of this section is to construct geometric examples which establish Theorem 1.3 .

We begin with the unit radius round metric on the sphere $S^{n}$. Now smoothly flatten a small neighbourhood of each of the poles, in such a way that the resulting metric is still rotationally symmetric about the north-south axis. Denote this metric by $g$. We can view $g$ as a warped product metric $g=d t^{2}+f^{2}(t) d s_{n-1}^{2}$ where the function $f:[\epsilon, \pi-\epsilon] \rightarrow[0,1]$ is given by $f(t)=t-\epsilon$ for $t \in[\epsilon, 2 \epsilon], f(t)=\pi-t-\epsilon$ for $t \in[\pi-2 \epsilon, \pi-\epsilon]$, and $f(t)=\sin t$ for $t \in[3 \epsilon, \pi-3 \epsilon]$ for some very small $\epsilon>0$. Over the remaining subintervals assume $f$ is defined in a way that results in it being everywhere smooth and concave down, and such that $f(t)=f(\pi-t)$ for all $t \in[\epsilon, \pi-\epsilon]$.

Note that the lines of longitude (that is, the $t$ parameter lines on $S^{n}$ ) are geodesics of $\left(S^{n}, g\right)$, and that the poles are mutually conjugate points.

Now consider a small round ball $B$ of some radius $R<<\pi / 2$ in the equator $S^{n-1} \subset S^{n}$. Note that the equator, (which corresponds to $t=\pi / 2$ ), is a unit round sphere. Finally let $S$ be the set of points in $S^{n}$ which lie on the longitudinal minimal geodesics joining the north to south poles which pass through $B$. Thus $S$ resembles a segment of an orange with flattened ends.

Take $n$ copies of $S$, and label these $S_{1}, \ldots, S_{n}$. We can form a union by identifying the south pole of the segment $S_{i}$ with the north pole of the segment $S_{i+1}$ for $i=1, \ldots, n-1$. Moreover, we can do this in such a way that the resulting singular manifold $X$ is smooth, in the sense that the longitudinal geodesics extend smoothly across the singular points where the poles have been glued. Considering a variation through such geodesics, it is immediate that the north pole of $S_{1} \subset X$ is conjugate to the other $n$ singular points.

The task is now to create from $X$ a genuine smooth Riemannian manifold-withboundary $M$ which has the same conjugate point property. To do this, focus on an $\epsilon / 2$ neighbourhood of any singular point in $X$. This neighbourhood either takes the form of a flat solid cone (at the two end points of $X$ ) or the union of two such cones at their cone points (at each intermediate singular point). Either way, we can embed these neighbourhoods isometrically into a flat ball of radius $\epsilon / 2$. The union of $X$ with all of these balls now contains a smooth 'strip' diffeomorphic (but not isometric) to the solid tube $[0,1] \times D^{n-1}$, incorporating all the original singular points, which retains the desired conjugate point property.

The manifold $M$ can now be embedded into any smooth manifold, irrespective of topology, and its metric extended so as to produce a smooth global Riemannian metric for which we can find (at least) $n$ points conjugate to some given point. A simple situation where there are precisely $n$ points conjugate to a given point can be created by embedding $M$ into $\mathbb{R}^{n}$. If we extend the metric on $M$ by first forming the union of $M$ with two flat, solid, infinite cones, which have cone points coincident with the original outer singular points of $X$, and cone angle not greater than the cone angles of the segment $S$. We do this in such a way that the longitudinal geodesics emerging from $M$ continue into the infinite cones. Away from the boundary (which in general will be non-smooth), the Riemannian metric on this union is smooth. Moreover the longitudinal geodesics, which leave $M$ in 
divergent fashion, will continue to diverge to infinity. Thus further conjugate points along any of these geodesics is prevented. By removing a small neighbourhood of the boundary, we obtain a new manifold with boundary diffeomorphic to the infinite solid tube $\mathbb{R} \times D^{n-1}$, which we embed smoothly into $\mathbb{R}^{n}$. Finally, extend the metric in any way to a smooth metric on the whole of $\mathbb{R}^{n}$.

For the case of infinitely many conjugate points in $\mathbb{R}^{n}$, we can repeat the above procedure starting with an infinite family of segments $\left\{S_{i}\right\}_{i=1}^{\infty}$, and subsequently forming a union with a single cone at the north pole of $S_{1}$. At the other extreme, any manifold with non-positive sectional curvature is well-known to have no conjugate points (see [doC, page 149]): for example $\mathbb{R}^{n}$ with its standard flat metric, or a flat torus $T^{n}$.

\section{$\S 4$ Derivatives of Jacobi solutions.}

Our aim in this section is to prove Theorem 1.4, which is a result analogous to Theorem 1.1 (though a little more restrictive) for the derivatives of solutions to $(J)$. It is easy to find examples of Jacobi equations of the type described by Theorem 1.4. For instance, consider again Example 2.12, in which $K \equiv-1$. Any solution to this Jacobi equation has the form $a e^{t}+b e^{-t}$ for some $a, b, \in \mathbb{R}$. A non-zero solution either has a derivative which vanishes exactly once (at the point $t=0.5 \ln (b / a)$ if $a, b>0$ or $a, b,<0$ ), or fails to vanish (if $a$ and $b$ have opposite signs, or one of them is zero).

Note that the behaviour of derivatives of Jacobi equations is not without geometric significance. For example, the vanishing of such derivatives is a crucial ingredient in the definition of focal points: in terms of the equation $(J)$, the point $t_{0} \in \mathbb{R}$ is said to be a focal point of $t_{1} \in \mathbb{R}$ if there is a solution $y(t)$ of $(J)$ such that $y\left(t_{1}\right)=1, y^{\prime}\left(t_{1}\right)=0$ and $y\left(t_{0}\right)=0$. More geometrically, a point $p$ in a Riemannian manifold $M$ is a focal point for a submanifold $N \subset M$ if, roughtly speaking, there is a one-parameter family of geodesics issuing normally from $N$ which all meet at the point $p$. For a precise definition see for example [doC; p230].

Before proving Theorem 1.4 we need a lemma, which is an analogue of Lemma 2.1 for the derivatives of Jacobi solutions.

Lemma 4.2. Suppose that $f(t)$ is a non-zero solution to a Jacobi equation $(J)$ for which $K$ is nowhere zero. If the derivative $f^{\prime}\left(t_{0}\right)=0$ for some $t_{0} \in \mathbb{R}$, then $f^{\prime}(t)$ changes sign through $t=t_{0}$.

Proof of 4.2: Suppose that $f^{\prime}(t)$ fails to change sign through $t=t_{0}$. The only way this can happen is if $f^{\prime}$ has a local maximum or minimum there, which implies that $f^{\prime \prime}\left(t_{0}\right)=0$. By $(J)$ we then have $K\left(t_{0}\right) y\left(t_{0}\right)=0$, and by our hypothesis $K\left(t_{0}\right) \neq 0$, so we conclude that $y\left(t_{0}\right)=0$. However, the conditions $y\left(t_{0}\right)=y^{\prime}\left(t_{0}\right)=0$ mean that $y$ is the zero solution, giving a contradiction. Thus $f^{\prime}$ must change sign through $t_{0}$.

Corollary 4.3. Suppose that $K$ is nowhere zero, and that every non-zero solution to $(J)$ has a derivative which vanishes precisely once. Let $f_{s}, s \in I$, be a continuous oneparameter family of non-zero solutions to $(J)$, and let $z_{s}^{\prime}$ be defined by $f_{s}^{\prime}\left(z_{s}^{\prime}\right)=0$. Then $z_{s}^{\prime}$ varies continuously with respect to $s$. 
Proof of 4.3: The proof is an easy analogue of the proof of Corollary 2.3 (and Lemma 2.2) with $f_{s}^{\prime}$ replacing $f_{s}$, and with Lemma 4.2 playing the role of Lemma 2.1.

Note that we need to impose conditions on $(J)$ in order for the conclusion of Corollary 4.3 to be true, as a smooth family of smooth functions each with a single zero need not have the zero varying continuously. For example consider the family of functions $f_{s}(t)$ with $s \in[0,1 / 10]$, where $f_{s}(t):=e^{t}-s$ for $t \leq 0$ and $f_{s}(t)=(1-s)(t-1)^{2}+t s$ for $t>0$. This is only $C^{0}$ at $t=0$, but can clearly be smoothed for each $s$ in a very small neighbourhood of $t=0$ to give a smooth path with all $f_{s}$ having a unique zero. However, this path of zeros is not continuous, as it has a discontinuity in the limit as $s \rightarrow 0^{+}$.

Proof of 1.4: We proceed by contradiction: suppose that the derivative of every solution of $(J)$ vanishes. As before, let $S$ denote the set of all solutions to $(J)$, and let $S^{\prime}:=\left\{y^{\prime} \mid y \in S\right\}$. Consider the function

$$
F: S \backslash\{0\} \rightarrow S^{\prime} \backslash\{0\} \times \mathbb{R}
$$

given by $F(y):=\left(y^{\prime}, z_{y}^{\prime}\right)$ where $z_{y}^{\prime}$ is the unique zero of $y^{\prime}$. Also consider the function

$$
G: S^{\prime} \backslash\{0\} \times \mathbb{R} \rightarrow \mathbb{R} \backslash\{0\}
$$

given by $G\left(\left(y^{\prime}, z_{y}^{\prime}\right)\right):=y\left(z_{y}^{\prime}\right)$. Note that $y\left(z_{y}^{\prime}\right) \neq 0$, since $y\left(z_{y}^{\prime}\right)=y^{\prime}\left(z_{y}^{\prime}\right)=0$ would force $y$ to be the zero solution. By Corollary 4.3, we see that both $F$ and $G$ are continuous functions, hence so is the composition

$$
G \circ F: S \backslash\{0\} \rightarrow \mathbb{R} \backslash\{0\} .
$$

Now $S \backslash\{0\} \cong \mathbb{R}^{2} \backslash\{0\}$, and is therefore a connected space. Observe that the image of $G \circ F$ lies in both components of $\mathbb{R} \backslash\{0\}$, since if $y$ is a solution of $(J)$ then so is $-y$. We therefore have a continuous map from a connected domain with a disconnected image, giving the desired contradiction.

\section{References}

[BBB] W. Ballman, M. Brin, K. Burns, On surfaces with no conjugate points, J. Diff. Geom. 25 (1987), 249-273.

[BK] K. Burns, G. Knieper, Rigidity of surfaces with no conjugate points, J. Diff. Geom. 34 (1991), 623-650.

[Ch] I. Chavel, Riemannian Geometry: A Modern Introduction, Cambridge Univ. Press, (1993).

[doC] M. do Carmo, Riemannian Geometry, Birkhäuser, 1992.

[G] R. Gulliver, On the variety of manifolds without conjugate points, Trans. Amer. Math. Soc. 210 (1975), 185-201.

[GG] L. Green, R. Gulliver, Planes without conjugate points, J. Diff. Geom. 22 (1985), 43-47. 
[H] P. Hartman, Ordinary Differential Equations, Classics in Applied Mathematics 38, SIAM (2002).

[W] D. J. Wraith, Ricci curvature decay on open manifolds, Bull. London Math. Soc. 35 no. 1 (2003), 72-78.

J.M. Burns and E. Staunton:

School of Mathematics, Statistics and Applied Mathematics,

National University of Ireland, Galway,

University Road, Galway,

Ireland.

D. J. Wraith,

Department of Mathematics and Statistics,

National University of Ireland, Maynooth,

Maynooth, County Kildare,

Ireland. 